\title{
The Impact of Site-Specific Bioconjugation on the Interfacial Activity of a Protein Biosurfactant
}

Hossam H. Tayeb $^{\mathrm{a} 1}$, Marina Stienecker ${ }^{\mathrm{a}}$, Anton P. J. Middelberg ${ }^{\mathrm{a} 2}$ and Frank Sainsbury ${ }^{\mathrm{a} 3 *}$

${ }^{a}$ The University of Queensland, Australian Institute for Bioengineering and Nanotechnology, St Lucia, QLD 4072, Australia.

Keywords:

Biosurfactants - Bioconjugation - PEGylation - Interfacial activity - Surface modification

\section{Corresponding author}

Frank Sainsbury

E-mail: f.sainsbury@griffith.edu.au

Tel: +61 737356066

\footnotetext{
${ }^{1}$ Present address: Faculty of Applied Medical Sciences, King Abdulaziz University, Jeddah 21589, Saudi Arabia

${ }^{2}$ Present address: Faculty of Engineering Computer \& Math Sciences, The University of Adelaide, Adelaide, SA 5005, Australia

${ }^{3}$ Present address: Griffith Institute for Drug Discovery, Griffith University, Nathan, QLD 4111, Australia
} 


\begin{abstract}
Biosurfactants, are surface active molecules that can be produced by renewable, industrially scalable biologic processes. DAMP4, a designer biosurfactant, enables the modification of interfaces via genetic or chemical fusion to functional moieties. However, bioconjugation of addressable amines introduces heterogeneity that limits the precision of functionalization as well as the resolution of interfacial characterization. Here we designed DAMP4 variants with cysteine point mutations to allow for site-specific bioconjugation. The DAMP4 variants were shown to retain the structural stability and interfacial activity characteristic of the parent molecule, while permitting efficient and specific conjugation of polyethylene glycol (PEG). PEGylation results in a considerable reduction on the interfacial activity of both single and double mutants. Comparison of conjugates with one or two conjugation sites shows that both the number of conjugates as well as the mass of conjugated material impacts the interfacial activity of DAMP4. As a result, the ability of DAMP4 variants with multiple PEG conjugates to impart colloidal stability on peptide-stabilized emulsions is reduced. We suggest that this is due to steric constraints on the structure of amphiphilic helices at the interface. Specific and efficient bioconjugation permits the exploration and investigation of the interfacial properties of designer protein biosurfactants with molecular precision. Our findings should therefore inform the design and modification of biosurfactants for their increasing use in industrial processes, and nutritional and pharmaceutical formulations.
\end{abstract}




\section{Introduction}

Self-assembling biosurfactants have been explored for use in different areas including food science, ${ }^{1-2}$ pharmaceuticals, ${ }^{3-5}$ cosmetics $^{6-7}$ and the petroleum industry. ${ }^{8}$ Biosurfactants offer many advantages when compared to chemically-synthesized equivalents such as biocompatibility, biodegradability, and sustainable manufacture. ${ }^{9-10}$ Interactions that drive the assembly of protein biosurfactants into supramolecular interfacial arrays are determined by the primary sequence, which dictates folding into $\alpha$-helices or $\beta$-sheets. Their interfacial activity is thereby defined by intermolecular and intramolecular electrostatic and hydrophobic interactions, as well as hydrogen bonds and van der Waals forces. Genetic or chemical modifications provide an opportunity to expand the functional repertoire of protein biosurfactants, but they must also consider the possibility of structural alteration and resulting biophysical implications on function.

DAMP4 is an $11.1 \mathrm{kDa}$ protein biosurfactant that was generated by linking four copies of a designer amphiphilic peptide into an anti-parallel four-helix bundle with a hydrophobic core. ${ }^{11}$ It is extraordinarily stable in bulk solution ${ }^{12}$ and amenable to industrially relevant purification procedures. ${ }^{9}$ Yet it is a highly functional surface-active molecule that has the capacity to completely reorganize its tertiary structure at the interface, resulting in a linear or planar amphiphilic polypeptide that greatly reduces interfacial tension of air-water and oil-water interfaces. As such, it is able to mediate switchable foaming under variable solution conditions ${ }^{13}$ and possesses the unique ability to integrate into interfacial films pre-stabilized by the helical monomers that constitute DAMP4. ${ }^{14}$ These features have seen DAMP4 used to modify oil-in-water emulsions providing functionality for inorganic shell deposition, ${ }^{15}$ immune-shielding, ${ }^{16}$ microparticle coating in drug delivery ${ }^{17}$ and cell-receptor targeting. ${ }^{16,18}$

We have previously shown that the extent of non-specific interactions between a DAMP4modified emulsion and the surrounding environment can be controlled by the mass and density of decorating polyethylene glycol (PEG) molecules. ${ }^{18}$ So called PEGylation is widely applied to improve the performance biopharmaceuticals and nanoparticles by reducing immunogenicity, and increasing serum half-life and stability. ${ }^{19-21}$ However, PEGylation of proteins has many challenges including polydispersity of PEG during chemical synthesis or inefficient reaction yield. ${ }^{22}$ Modification of DAMP4 using N-hydroxysuccinimidyl (NHS)functionalized PEG yields heterogeneous reactions products resulting in limitations on the physico-chemical characterization of DAMP4-populated interfaces as well as on the precision 
of surface modification. ${ }^{18}$ However, bioconjugation chemistries offer a range of possible modifications dependent on the accessibility of suitable amino acid side chains. ${ }^{23}$ For example, conjugates can be coupled to proteins through functional groups including hydroxyl, carboxyl, and thiol-reactive compounds. Thiol-maleimide chemistry is widely applied to obtain sitespecific bioconjugation ${ }^{24}$ as the less frequent occurrence of solvent-exposed cysteine residues often allows for their rational insertion by site-specific mutagenesis.

Bioconjugation should impart the desired modification on a biomolecule whilst preserving its activity. In the case of biosurfactants, function is achieved via adsorption at an interface between two phases. This generally occurs in two steps; transfer or transportation of the protein molecule to the interface and protein unfolding and rearrangement at the interface. ${ }^{25}$ Tailorable nanocarrier emulsions (TNEs) are peptide-stabilized oil-in-water $(\mathrm{O} / \mathrm{W})$ emulsions that can be functionalized by the spontaneous integration of genetically or chemically modified versions of DAMP4. ${ }^{16,26}$ During the assembly of TNEs with multifunctional interfaces, DAMP4 conjugated to polyethylene glycol (PEG) is used to provide colloidal stability during additional surface functionalization. Here we genetically engineered DAMP4 to improve the accuracy with which it can be used to impart functionality to the surface of peptide-stabilized emulsions, such as PEGylation. The rational introduction of cysteine residues at select positions designed to retain structure and function of DAMP4 resulted in highly efficient site-specific bioconjugation using the maleimide-thiol reaction. We report the impact of single and double cysteine insertions on interfacial activity and address the question of whether the number of conjugates or the total mass of conjugated polymer affect this parameter. This work shows how genetic and chemical modification of amphiphilic structural features relates to the design of functional interfaces stabilized by biosurfactants. 


\section{Materials and methods}

\section{Molecular cloning}

DAMP4-encoding sequences were ordered from Integrated DNA Technologies (www.idtdna.com) with extensions homologous to the flanking insertion site of the E.coli expression vector pET48b+ (Novagen; www.merckmillipore.com). Coding sequences were amplified with upstream (5`-ACTTTAAGAAGGAGATATACAT-3`) and downstream (5`TGTACAGAATTCGGATCC-3`) oligonucleotides corresponding to the extensions and the vector was amplified with their reverse complements. Expression constructs were assembled using the NEBuilder HiFi DNA assembly master mix (New England Biolabls; www.neb.com) and clones were verified by Sanger sequencing.

\section{Expression and purification of DAMP4 variants}

DAMP4 variants were expressed and purified in E.coli as previously described except where indicated [13]. Briefly, each variant was purified using sequential chromatography steps; immobilized metal affinity chromatography by taking advantage of the presence of eight histidine residues in each protein sequence, ion exchange chromatography at $\mathrm{pH} 7.0$ and reversed-phase (RP) HPLC. Purified proteins were lyophilized and analyzed for purity and concentration using analytical HPLC against DAMP4 standard curve. Accurate concentrations were determined by amino acid analysis conducted by the Australian Proteome Analysis Facility (APAF).

\section{PEGylation of DAMP4 variants}

DAMP4 variants were resuspended with 25 mM HEPES (pH 7.0), 2 mM TCEP and PEGylated using $5 \mathrm{kDa}$ maleimide-functionalized methoxy polyethylene glycol (www.sigmaaldrich.com). PEGylation of DAMP4 variants was carried out at $4{ }^{\circ} \mathrm{C}$ for approximately $16 \mathrm{~h}$. The PEGylation reaction for all DAMP4 variants was evaluated by SDS-PAGE using the Any-kD TGX Precast Protein Gels (www.bio-rad.com).

\section{Interfacial tension analysis}

The interfacial tension (IFT) kinetics for the DAMP4 variants were measured using a DSA-10 droplet-shape analysis unit (www.kruss.de). The sample volume of each protein $(10 \mu \mathrm{M})$ is 8 
$\mathrm{ml}$ in 25 mM HEPES ( $\mathrm{pH}$ 7.0) loaded onto a quartz cuvette (www.hellma-analytics.com). Approximately $10 \mu$ Miglyol $^{\circledR} 812$ (www.axoindustries.be) droplets were manually formed using a known-diameter U-shaped stainless steel capillary fed by a glass syringe. The obtained interfacial tension was based on the Young-Laplace equation through images captured by a connected camera for each oil droplet at an approximate rate of 1 measurement/second. The surface tension of a mature pristine oil droplet was $31 \mathrm{mN} / \mathrm{m}$ and IFT values were derived by averaging the final $100 \mathrm{sec}$ of measurement.

\section{Circular dichroism spectroscopy}

To analyze the secondary structure (Helicity level) of each DAMP4 variant, at $0.25 \mathrm{mg} / \mathrm{ml}$ in 2.8 mM HEPES (pH 7.0), 2 mM tris(2-carboxyethyl)phosphine (TCEP), we determined the CD spectra using (http://www.jascoinc.com) at 190-260 nm. CD spectra measurements (300 s) were conducted in a $1 \mathrm{~mm}$ path length glass cuvette (www.starnacells.com) at 20 and 90 ${ }^{\circ} \mathrm{C}$ to evaluate the stability against high temperature for all DAMP4 variants. Molar ellipticity was derived from collected data of each variant.

\section{Differential scanning calorimetry (DSC)}

DSC analysis was performed to evaluate the thermal stability of the DAMP4 mutants before and after PEGylation. The experiment was conducted using the MicroCal VP- DSC Microcalorimeter (http://www3.gehealthcare.com) and the VPViewer 2000 DSC software. The test and reference samples were treated using the ThermoVac degassing station at $25^{\circ} \mathrm{C}$ for 5 minutes to eliminate air bubbles. The molar ratio of DAMP4 mutant samples $(0.75 \mathrm{mg} / \mathrm{ml})$ to TCEP was maintained at 1:10. DSC measurements were conducted at scan rate ranging from $25{ }^{\circ} \mathrm{C}$ to $125{ }^{\circ} \mathrm{C}$. The collected data were analyzed using the MicroCal-enabled Origin data analysis package.

\section{Preparation and characterization of TNEs}

Lyophilized AM1 (molar mass 2473 Da, $\geq 95 \%$ purity) was custom synthesised by Genscript (www.genscript.com) and dissolved using 25 mM HEPES (pH 7.0) containing $800 \mu \mathrm{M}$ of $\mathrm{ZnCl} 2$, to reach a final concentration of $400 \mu \mathrm{M}$. Miglyol was added to the AM1 solution to a final oil composition of $2 \% \mathrm{v} / \mathrm{v}$. The mixture was sonicated four times at $45 \mathrm{~s}$ using the Branson Sonifier 450 Ultrasonicator (www.emersonindustrial.com). AM1-stabilised emulsions were 
added drop-by-drop onto to each PEGylated DAMP4 variant (100 $\mu \mathrm{M})$ while vigorously stirring in a 1:1 volumetric ratio. Prepared TNEs were analyzed using dynamic light scattering and zeta potential (www.malvern.com).

For stability analysis the concentration used of each PEGylated DAMP4 variant was $10 \mu \mathrm{M}$. TNEs modified by PEG-S28C and PEG-D4C2 were diluted in 25 mM HEPES (pH 7.0) or 0.1 M EDTA in the same buffer at 1:1. Emulsions were labelled with 1,1'-Dioctadecyl- 3,3,3 03 0 -tetramethylindocarbocyanine perchlorate (DiI; www. thermofisher.com) and monitored over time to evaluate TNEs stability. 


\section{Results and discussion}

\section{DAMP4 variants}

The protein biosurfactant, DAMP4 (11.1 kDa), is composed of four repeats of the helical amphiphilic peptide AM1 (Figure 1A). Each repeat is linked by a short sequence designed to break the helix and provide the conformational flexibility that allows folding into a 4-helix bundle structure. ${ }^{11}$ In order to insert unique sites for efficient bioconjugation, cysteine mutations were generated by replacing serine residues within the linkers. Selecting this location should not affect the structure and interfacial activity of DAMP4 given the similarity between the substituted side chains. In addition, the C99 variant was created by adding a cysteine residue to the carboxy (C)-terminus of DAMP4 (Figure 1B). All variants were successfully expressed and purified (Figure S1).

A

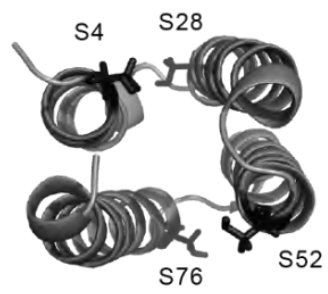

B

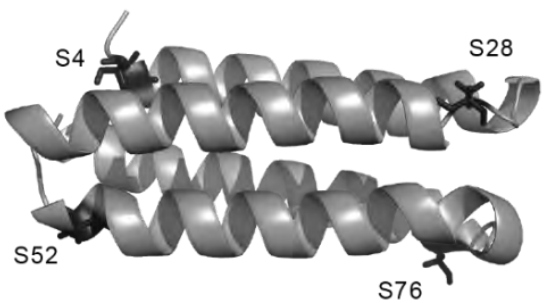

C

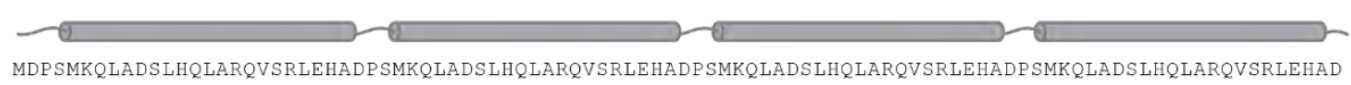

DAMP4 MDPSMKQLADSLHQLARQVSRLEHADPSMKQLADSLHQLARQVSRLEHADPSMKQLADSLHQLARQVSRLEHADPSMKQLADSLHQLARQVSRLEHAD

$\mathrm{S} 4 \mathrm{C}$

$\mathrm{S} 28 \mathrm{C}$

$\mathrm{S} 52 \mathrm{C}$

C99

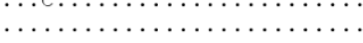

(1)

.

Figure 1. Sequence design for DAMP4 cysteine variants. End (A) and side (B) views of a molecular model of the four-helix bundle DAMP4 protein with mutated serine residues indicated. (C) The sequence variation between single cysteine mutants developed in this study. Dots represent conserved residues.

\section{Structure, stability and activity of cysteine mutants}

DAMP4 is an extremely stable four-helix bundle in solution ${ }^{9}$ and its helicity is preserved at high temperature. ${ }^{12}$ However, it is possible that even rational modifications to the sequence might interfere with the structure or function of DAMP4. Therefore, to investigate the structure and stability of the cysteine variants we performed CD spectroscopy. At $20{ }^{\circ} \mathrm{C}$, all single cysteine mutants showed similar CD spectra to DAMP4, characterized by the double 
absorption minima at $222 \mathrm{~nm}$ and $208 \mathrm{~nm}$ (Figure S2). This indicates that all variants show a similar level of helicity. Thermal stability was also performed by CD spectroscopy for each protein at $90{ }^{\circ} \mathrm{C}$, which showed that the variants retained the remarkable stability of DAMP4 (Figure 2A).

A

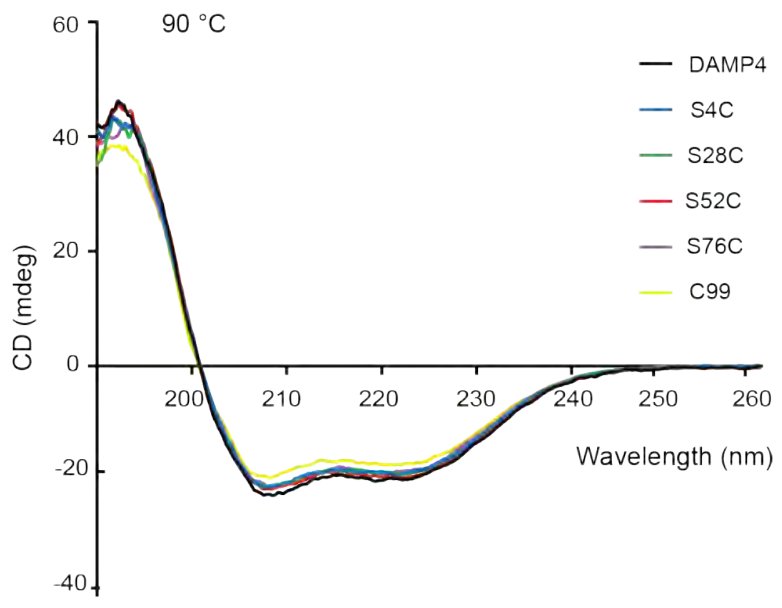

B

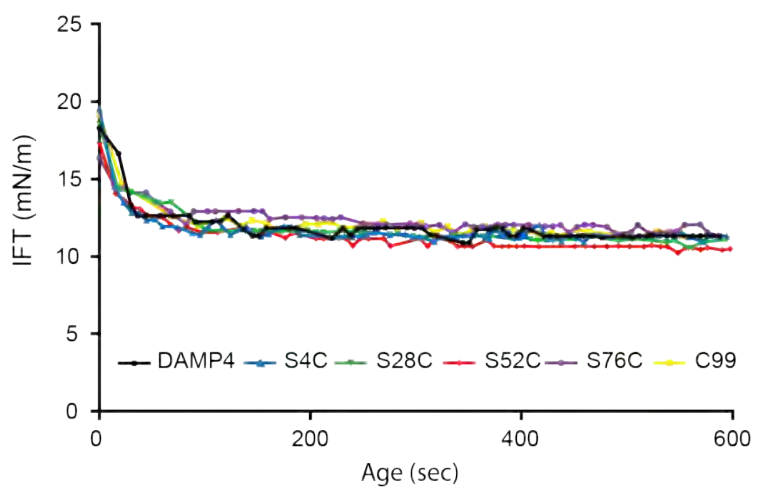

Figure 2. Thermal stability and interfacial activity of DAMP4 single cysteine variants. (A) CD spectra of DAMP4 variants at $90^{\circ} \mathrm{C}$. (B) Interfacial tension analysis of DAMP4 variants.

To evaluate the impact of the mutations on the interfacial activity of each DAMP4 variant, we determined interfacial tension of an $\mathrm{O} / \mathrm{W}$ droplet in the presence of the biosurfactants. Interfacial tension in the presence of DAMP4 shows an immediate reduction, levelling off to 11.32 Nm/m consistent with the adsorption of the protein at the interface. ${ }^{18}$ The variants all resulted in a similar surface tension reduction, indicating that all DAMP4 variants possess similar interfacial activity (Figure 2B). This is expected as the interfacial activity of DAMP4 is governed by the helicity of AM1 motifs present in both the four-helix bundle in solution and the unfolded protein at the $\mathrm{O} / \mathrm{W}$ interface. ${ }^{11,} 14$ This shows that our rational genetic modification/substitution with cysteine residues does not have an effect on the overall 
interfacial activity of DAMP4. Preserving the interfacial activity for DAMP4 variants allows their further use in the stabilization and functionalization of TNEs.

\section{Functional bioconjugation to DAMP4 variants}

The single cysteine mutants were genetically engineered through the substitution/addition of cysteine residues to obtain site-specific bioconjugation. In previous work we showed that the density and mass of PEG molecules presented on the surface of nanoscale emulsions was central to the properties of the interface and accessibility of co-presented functional moieties. ${ }^{18}$ However, the PEGylation was variable and resulted in a herterogenous population of PEGylated DAMP4. Here we PEGylated each DAMP4 variant using maleimide-functionalized methoxy PEG (5 kDa). The efficiency of the PEGylation reaction was evaluated using SDSPAGE and showed successful PEGylation at a yield approaching 100\% (Figure S3). The precise and efficient modification of DAMP4-derived biosurfactants with maleimide-thiol chemistry is a significant improvement on the variable amine labelling previously employed. ${ }^{18}$ Such defined conjugates will enable controlled evaluation of the structure and function of DAMP4-modified interfaces.

DAMP4 facilitates the presentation of a wide range of biomolecules via the spontaneous integration onto an AM1-stabilised interface through an as yet undefined mechanism (Figure 3A). To test the interfacial activity of PEG conjugates of the DAMP4 variants, we assessed their ability to impart colloidal stability on AM1-stabilised emulsions. The positive surface charge of individual droplets results in a net repulsion effect that prevents coalescence on the timescale of days. However, dilution of the peptide-stabilized emulsion into a high salt solution negates the positive charge leading to rapid coalescence in the absence of a stabilizing modification. The adsorption of PEGylated DAMP4 onto the TNE interface was thus confirmed by comparing them to the AM1 stabilized emulsion in PBS. While AM1-emulsions rapidly coalesce, TNEs stabilized with PEGylated DAMP4 mutants remain stable (Table S1). PEGylated DAMP4 variants were used at a final surface density of $14 \mathrm{pmol} / \mathrm{cm}^{2}$, which is well below the capacity for DAMP4 integration. ${ }^{18}$ The presentation of each PEGylated DAMP4 variant resulted in a slight increase in the droplet size when compared to AM1-emulsions (Figure 3B), which could be attributed to an increased hydrodynamic diameter of TNEs with PEG presented into the aqueous phase. ${ }^{18}$ Furthermore, PEG presentation decreased the zeta potential of TNEs following the integration of each PEGylated DAMP4 variant (Figure 3B) due in part to the slightly negative charge of DAMP4, though mostly to the effect of PEG 
shielding surface charge. Interestingly, the drop in zeta potential was variable even though each protein carries a single PEG. This suggests that the orientation and/or conformation of the PEG is affected by its position in DAMP4 and, therefore, the orientation of the cysteine insertions with respect to the interface. Future studies involving PEG orientation and architecture when presented on TNE interfaces are required. It is also not known how PEGylated DAMP4 interacts with AM1 during sequential adsorption onto the interface; what the role of PEG is, nor whether AM1 is displaced. Experiments to address these questions are now enabled by the specifically modifiable biosurfactants reported herein.
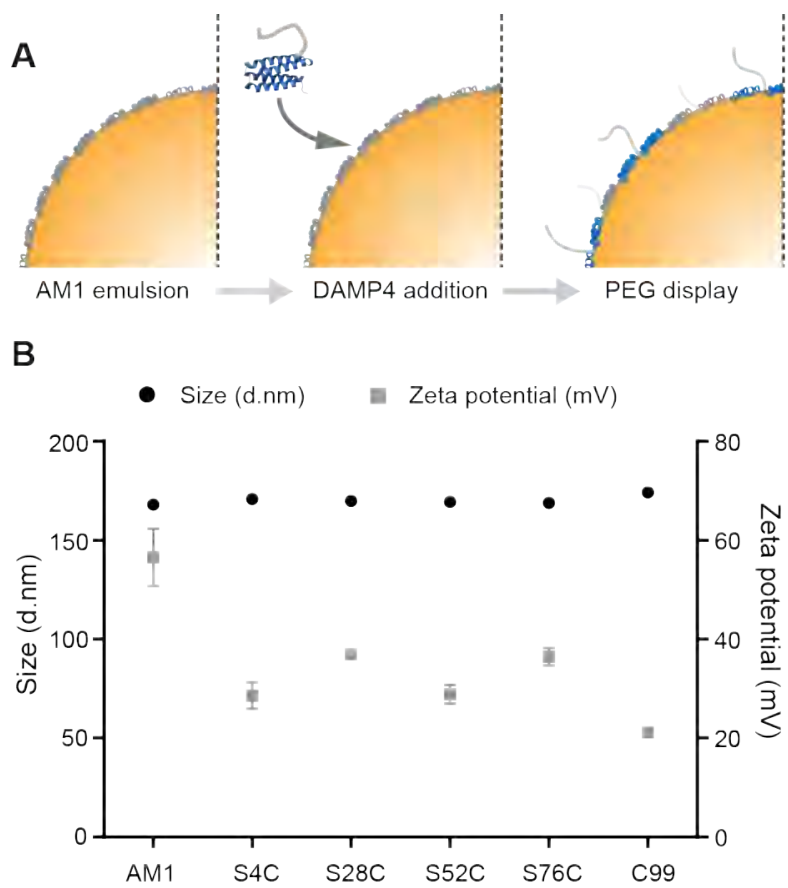

Figure 3. Functionalisation of TNEs by PEGylated DAMP4 variants. (A) Schematic representation of the spontaneous integration of DAMP4 onto the surface of an AM1-stabilised Miglyol emulsion. (B) DLS and zeta potential measurements of the TNEs modified with PEGylated DAMP4 cysteine mutants. TNEs size and zeta potential measurements are presented as black circles and grey squares respectively and values represent the average of three independent assembly steps.

\section{Stability, interfacial activity and bioconjugation to a double cysteine variant}

Devising an emulsion platform with controlled variability in PEG density gives maximum flexibility in interface design and will lead to greater understanding of the interactions between soft matter interfaces and the surrounding environment. Therefore we designed a DAMP4 variant that combines two of the cysteine insertions to enable the comparison of TNE formulations with varied PEG densities. Combining the S28C and S76C mutations created D4C2, with cysteines positioned at one end of the four-helix bundle (Figure 1A). We also 
cloned a variant that combined the S28C and S52C mutations, at opposing ends of the fourhelix bundle, but did not pursue this variant due to low level expression and a strong propensity to aggregate, even in the presence of high concentrations of reducing agents $(>0.1 \mathrm{M}$ dithiothreitol).

D4C2 is also predominantly helical although it was shown to have less pronounced $222 \mathrm{~nm}$ and $208 \mathrm{~nm}$ CD spectroscopy minima compared to DAMP4 (Figure 4A). Nevertheless, the helicity is preserved at $90{ }^{\circ} \mathrm{C}$ indicating that the double variant maintains high thermal stability. Despite the apparent decrease in helicity of D4C2, the interfacial activity at an O/W interface is similar to the constituent single mutants and DAMP4 (Figure 4B). It is possible that interaction of the amphiphilic helices with the oil phase imposes a constraint on helical conformation, reinforcing the same structural feature that imparts interfacial activity. In any case, the IFT results of D4C2 shows that the slight changes in the level of helicity did not interfere with its spontaneous integration onto an $\mathrm{O} / \mathrm{W}$ interface, which also suggests that it could also be used to modify an AM1-stabilised emulsion.
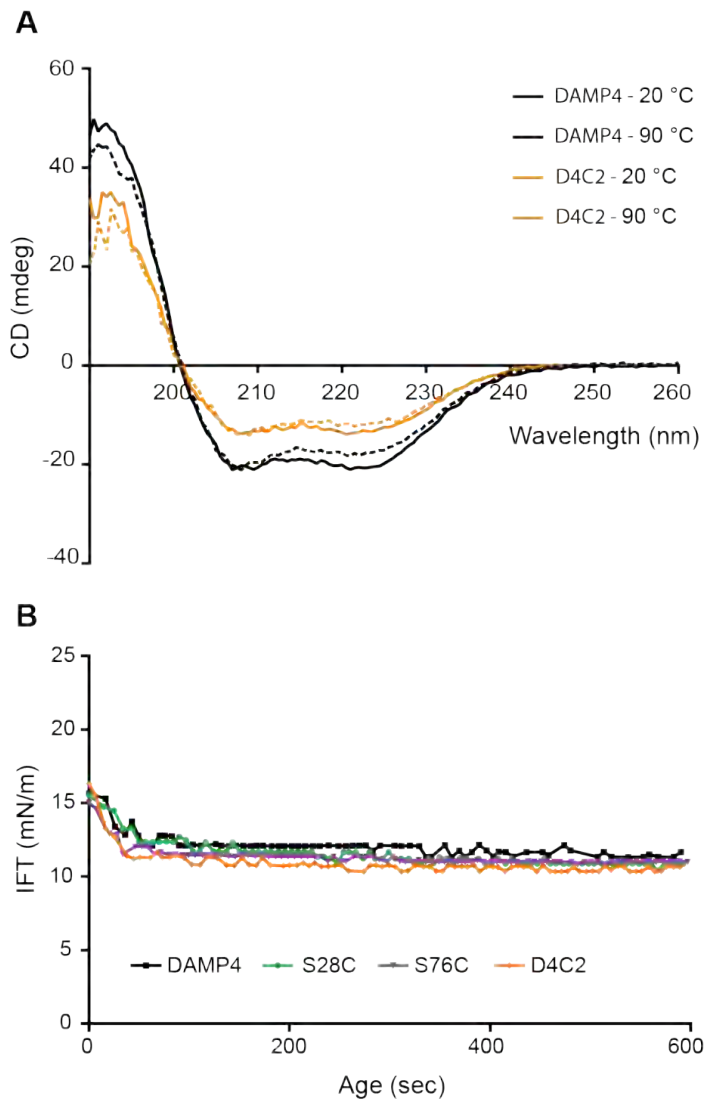

Figure 4. Thermal stability and interfacial activity of D4C2, a double cysteine variant of DAMP4. (A) CD spectra of DAMP4 and D4C2 recorded at both $20^{\circ} \mathrm{C}$ and $90^{\circ} \mathrm{C}$. (B) Interfacial tension analysis of D4C2 and variants possessing its constituent single mutations compared to DAMP4. 
SDS-PAGE separation of PEGylation reactions showed that bioconjugation via maleimidethiol chemistry also efficiently labels the thiols of both inserted cysteines of D4C2 (Figure 5A). We have previously shown by mass spectrometry that the additional molecular weight of the conjugates conferred by PEG appears to be 2-fold higher than expected on a polyacrylamide gel. ${ }^{18}$ Thus, the single and double cysteine mutants are conjugated to 1 and 2 PEGs, respectively. We investigated the size and charge of TNEs stabilized with PEGylated D4C2, S28C and S76C to evaluate the influence of different PEG densities on the interface. Again, the adsorption of each PEGylated variant of S28C, S76C and D4C2 was confirmed by TNE stability in PBS, which showed that while PEGylated D4C2 was able to stabilize TNEs there was a slight increase in hydrodynamic radius (Table S2). This was mirrored in the size variability among replicate emulsions stabilized by this conjugate (Figure 5B). Despite the presumed two-fold increase in PEG at the interface, there are no significant surface charge differences between the TNEs formulated with the single and double PEGylated DAMP4 variants (Figure 5B). This suggested reduced adsorption and that interfacial activity of DAMP4 variant could be differentially altered by PEGylation.
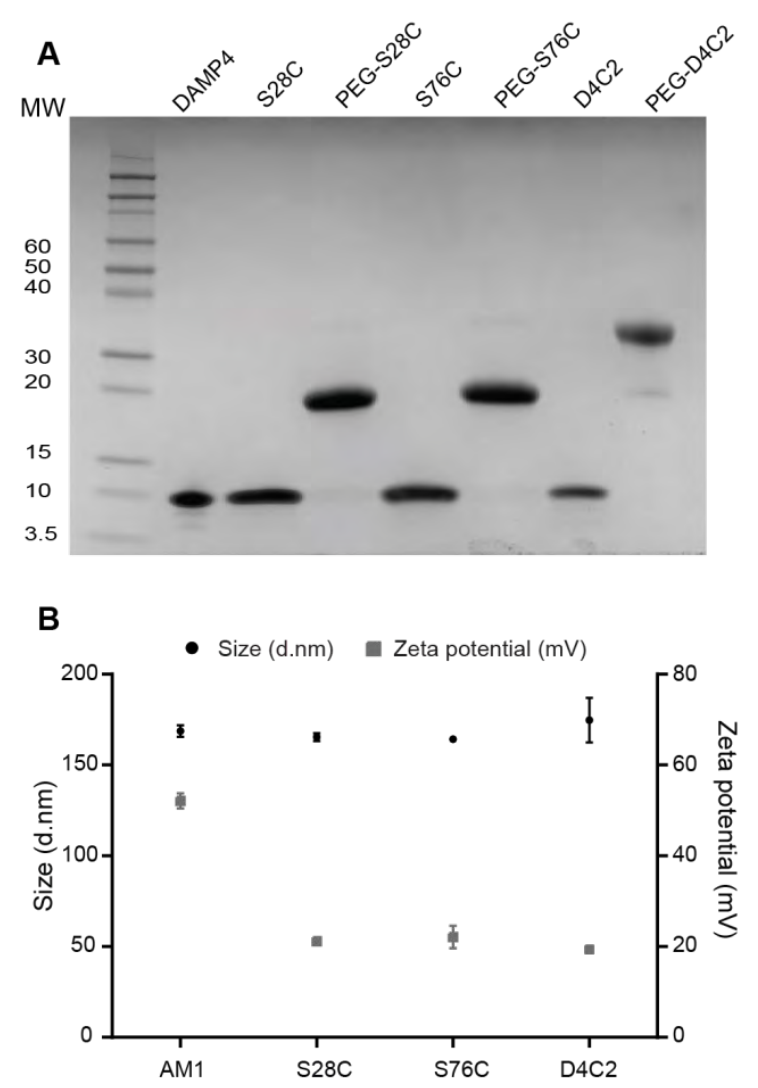
Figure 5. PEGylation and TNE functionalization by D4C2. (A) SDS-PAGE analysis of PEGylated D4C2 and its constituent single mutations. (B) DLS and zeta potential of TNEs formulated with these variants.

\section{Interfacial activity is affected by both the mass and number of conjugates}

Conjugation of the relatively large PEG molecules to DAMP4 is expected to have a significant impact on the kinetic mass transfer and, therefore, adsorption rate of DAMP4. Nevertheless, how the mass and position of the conjugate impacts steady-state interfacial activity is unknown and highly relevant to the design and deployment of tailored interfaces. Comparing the single (S28C) and double (D4C2) cysteine variants with either $5 \mathrm{kDa}(5 \mathrm{~K})$ or $10 \mathrm{kDa}(10 \mathrm{~K})$ conjugated PEG, revealed that not only does the mass of the conjugate impact interfacial activity, so too does the number of conjugates (Figure 6). To rule out an effect of PEG on the unfolding of DAMP4, which is necessary for its adsorption onto the interface, we subjected the PEGylated variants to differential scanning calorimetry. The transition temperature of both S28C and D4C2 were found to be unaffected by the conjugated PEG (Figure S4), indicating that the polymer has negligible impact on the stability of the proteins and, therefore, its capacity to unfold at the interface. While S28C-5K-PEG reduces IFT from the $31 \mathrm{mN} / \mathrm{m}$ of a pristine oil droplet to $15.8 \mathrm{mN} / \mathrm{m}$, S28C-10K-PEG only reduces IFT to $17.2 \mathrm{mN} / \mathrm{m}$. In comparison, the unconjugated protein reduces IFT to $11.1 \mathrm{mN} / \mathrm{m}$. PEGylated D4C2 reduced IFT to 19.3 and $21.9 \mathrm{mN} / \mathrm{m}$ when conjugated to $5 \mathrm{~K}$ and $10 \mathrm{~K}$ PEG, respectively. This additional insight afforded by efficient, site-specific bioconjugation enables us to refine our previous interpretation using heterogeneous conjugate mixtures, which suggested a negligible impact of conjugate mass. ${ }^{18}$ Here we show that the mass of the conjugate has a considerable impact on IFT, as does the number of conjugates. Comparison of D4C2-5K PEG (19.3 mN/m) with S28C-10K-PEG (17.2 $\mathrm{mN} / \mathrm{m}$ ), which possess the same theoretical mass of polymer and show that the number of PEGs has a considerable impact on IFT. This result highlights the utility of the DAMP4 variants and their precise conjugation in enabling the fundamental study of biosurfactants and their interfacial properties. 


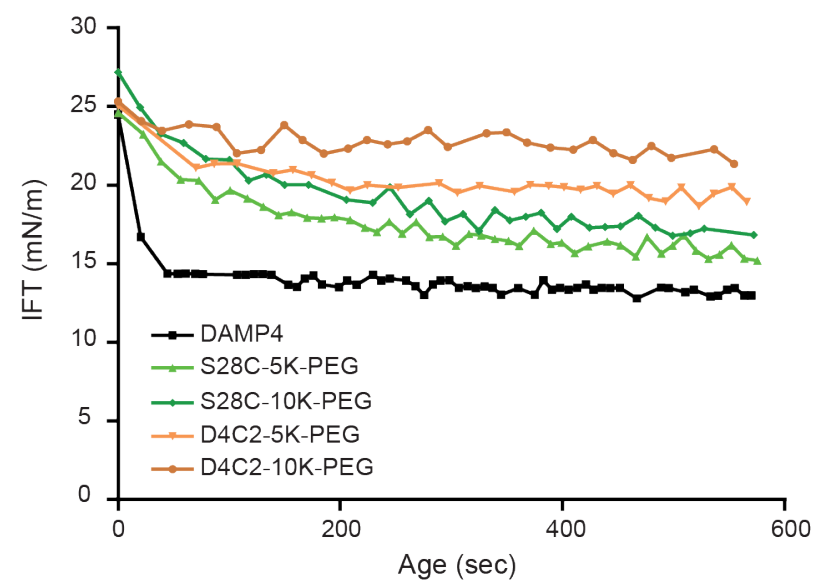

Figure 6. Interfacial activity of double and single PEGylated DAMP4. Analysis was performed on D4C2 and the single cysteine variant S28C conjugated to 5K and 10K PEG compared to DAMP4.

To examine the functional impact of the interference with interfacial activity due to PEG conjugation, we assembled TNEs with a low $\left(1.4 \mathrm{pmol} / \mathrm{cm}^{2}\right)$ density of PEG-DAMP4. Incubation with 0.1 M EDTA disrupts the cohesive interfacial peptide film by chelating the $\mathrm{Zn}^{2+}$ that acts to cross-link histidine-bearing AM1 peptides, resulting in susceptibility to coalescence. ${ }^{18,27}$ Using a low density of PEG-DAMP4 variant ensures that TNEs will coalesce within a reasonable timeframe. TNEs decorated with PEGylated S28C remained stable at least for 1 hour (Figure 7). In contrast, and despite the two-fold increase in PEG density, phase separation of the PEGylated D4C2 stabilized TNEs was detectable at approximately 2 min, similar to the undecorated AM1 emulsion (Figure 7). Thus, the differences in the interfacial activity of PEGylated S28C and D4C2 result in a functional impact on their ability to stably decorate TNEs. As the conjugate does not appear to affect the unfolding of DAMP4, these results suggest rather that the PEG conjugate can alter the conformation of interfacial DAMP4 or indeed directly interact with the interface. The influence of PEG on the hydrophobicity of proteins has been documented and increased PEG length increases hydrophobic interactions. ${ }^{28}$ Our contrary observations lead us to an alternative explanation; that the increase in PEG density presents a steric constraint on the permissible density of DAMP4 at the interface, effectively preventing adsorption. Indeed, we have previously found evidence to suggest the presence of PEG directly at the interface. ${ }^{18}$ However, we cannot rule out the possibility that electrostatic interactions between the AM1 interface and DAMP4 are important for the integration of DAMP4 and that such interactions could be masked by the increased mass of PEG, thereby reducing the adsorption of the more highly PEGylated variant. 


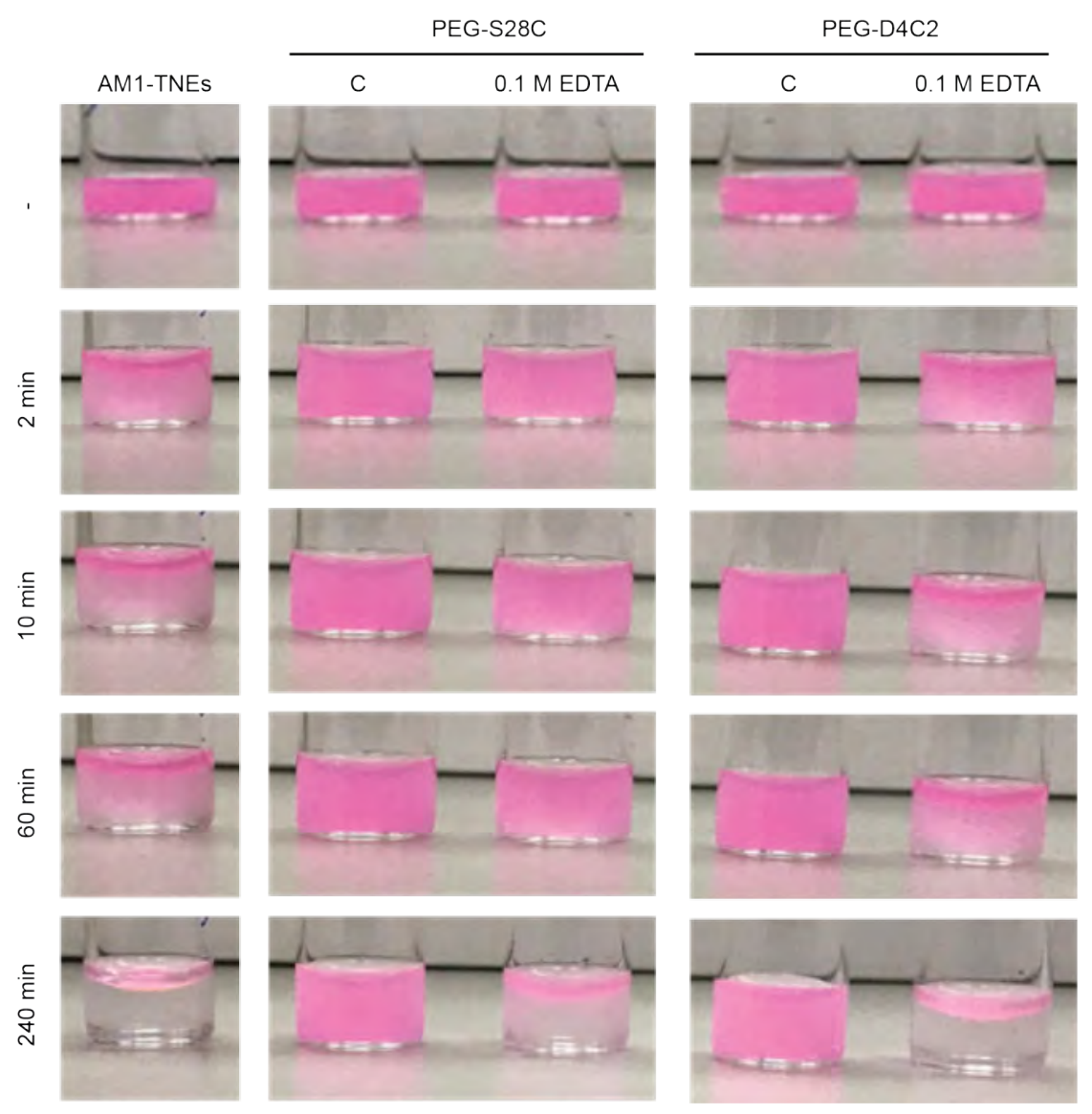

Figure 7. Stability of TNEs modified with PEGylated DAMP4 variants in the presence of EDTA. AM1 emulsions labelled with DiI, and those subsequently modified with PEG-S28C and PEG-D4C2 were diluted 1:1 in $25 \mathrm{mM}$ HEPES ( $\mathrm{pH} 7.0$ ) as a control (C) or the same buffer with a final EDTA concentration of $50 \mathrm{mM}$, and monitored for stability over time.

\section{Conclusion}

Controlling the interfacial properties of nanomaterials is essential for understanding their interactions with the surrounding environment. Here we used a bioengineering approach to obtain biosurfactants amenable to site-specific bioconjugation. Rational design of cysteine mutants of the designer protein surfactant, DAMP4, yielded variants with preserved structure, stability and interfacial activity that could be specifically PEGylated with yield approaching $100 \%$. The biologically produced DAMP4 has potential as a renewable surfactant in industrial processes and as a co-surfactant useful in the top-down functionalization of sophisticated interfaces for high-end applications. This work demonstrates that the primary sequence can be tailored for specific applications requiring chemical modification and will, for example, allow more precise functionalization of TNEs than was previously possible. Furthermore, controlled 
modification of these versatile biosurfactants will provide tools to investigate the fundamental relationship between structure and function of polypeptides at an interface. As an example, we showed that the number of conjugates as well as the mass of a conjugated polymer, PEG, influences interfacial activity, likely via steric constraints on the interaction of the biosurfactants with the $\mathrm{O} / \mathrm{W}$ interface. We expect that the outcomes of this study will provide the tools to further explore the design of functional interfaces and increase understanding of the relationship between the genetic and chemical modification of polypeptides and their function.

\section{Acknowledgments}

This work was supported by the University of Queensland ECR grant to FS. HHT acknowledges the financial support from the King Abdul-Aziz University, Ministry of Higher education, Saudi Arabia. Elements of this research utilized equipment and support provided by the QLD node of the National Biologics Facility (www.nationalbiologicsfacility.com), an initiative of the Australian Government being conducted as part of the NCRIS National Research Infrastructure for Australia. We also acknowledge the contribution of APAF staff and that the amino acid analysis was facilitated using the infrastructure provided by the Australian Government through the National Collaborative Research Infrastructure Strategy (NCRIS).

\section{Supporting Information}

SDS-PAGE analysis of purified DAMP4 variants (Figure S1); CD Spectroscopy of single cysteine mutants at $20^{\circ} \mathrm{C}$ (Figure S2); SDS-PAGE analysis of PEGylation (Figure S3); Stability measurements TNEs formulated with PEGylated DAMP4 single cysteine variants (Table S1); Stability measurements TNEs formulated with PEGylated DAMP4 double cysteine variants (Table S2); Stability of PEGylated DAMP4 variants in solution (Figure S4). 


\section{References}

1. McClements, D. J., Nanoscale nutrient delivery systems for food applications: improving bioactive dispersibility, stability, and bioavailability. J. Food Sci. 2015, 80 , N1602N1611.

2. McClements, D. J.; Rao, J., Food-grade nanoemulsions: formulation, fabrication, properties, performance, biological fate, and potential toxicity. Crit. Rev. Food Sci. Nutr. 2011, 51 , 285-330.

3. Gudiña, E. J.; Rangarajan, V.; Sen, R.; Rodrigues, L. R., Potential therapeutic applications of biosurfactants. Trends Pharmacol. Sci. 2013, 34 , 667-675.

4. Rodrigues, L.; Banat, I. M.; Teixeira, J.; Oliveira, R., Biosurfactants: potential applications in medicine. J. Antimicrob. Chemother. 2006, 57 , 609-618.

5. Tayeb, H. H.; Sainsbury, F., Nanoemulsions in drug delivery: formulation to medical application. Nanomedicine (London, U. K.) 2018, 13 , 2507-2525.

6. Varvaresou, A.; Iakovou, K., Biosurfactants in cosmetics and biopharmaceuticals. Lett. Appl. Microbiol. 2015, 61 , 214-23.

7. Vecino, X.; Cruz, J. M.; Moldes, A. B.; Rodrigues, L. R., Biosurfactants in cosmetic formulations: trends and challenges. Crit. Rev. Biotechnol. 2017, 37 , 911-923.

8. Perfumo, A.; Rancich, I.; Banat, I. M., Possibilities and challenges for biosurfactants use in petroleum industry. Adv. Exp. Med. Biol. 2010, 672, 135-45.

9. Dimitrijev Dwyer, M.; Brech, M.; Yu, L.; Middelberg, A. P. J., Intensified expression and purification of a recombinant biosurfactant protein. Chem. Eng. Sci. 2014, 105, 12-21.

10. Marchant, R.; Banat, I. M., Microbial biosurfactants: challenges and opportunities for future exploitation. Trends Biotechnol. 2012, 30, 558-565.

11. Middelberg, A. P. J.; Dimitrijev-Dwyer, M., A Designed Biosurfactant Protein for Switchable Foam Control. ChemPhysChem 2011, 12, 1426-1429.

12. Schaller, A.; Connors, N. K.; Dwyer, M. D.; Oelmeier, S. A.; Hubbuch, J.; Middelberg, A. P. J., Computational study of elements of stability of a four-helix bundle protein biosurfactant. J. Comput.-Aided Mol. Des. 2015, 29, 47-58.

13. Dimitrijev-Dwyer, M.; He, L.; James, M.; Nelson, A.; Wang, L.; Middelberg, A. P. J., The effects of acid hydrolysis on protein biosurfactant molecular, interfacial, and foam properties: pH responsive protein hydrolysates. Soft Matter 2012, 8, 5131-5139.

14. Dwyer, M. D.; He, L. Z.; James, M.; Nelson, A.; Middelberg, A. P. J., Insights into the role of protein molecule size and structure on interfacial properties using designed sequences. J. R. Soc., Interface 2013, 10, 20120987.

15. Wibowo, D.; Zhao, C.-X.; Middelberg, A. P. J., Interfacial Biomimetic Synthesis of Silica Nanocapsules Using a Recombinant Catalytic Modular Protein. Langmuir 2015, 31, 19992007.

16. Zeng, B.; Chuan, Y.; O'Sullivan, B.; Caminschi, I.; Lahoud, M.; Thomas, R.; Middelberg, A., Receptor-Specific Delivery of Protein Antigen to Dendritic Cells by a Nanoemulsion Formed Using Top-Down Non-Covalent Click Self-Assembly. Small 2013, 9, 3736-3742.

17. Yamada, M.; Tayeb, H.; Wang, H. Q.; Dang, N.; Mohammed, Y. H.; Osseiran, S.; Belt, P. J.; Roberts, M. S.; Evans, C. L.; Sainsbury, F.; Prow, T. W., Using elongated microparticles to enhance tailorable nanoemulsion delivery in excised human skin and volunteers. J. Controlled Release 2018, 288, 264-276.

18. Tayeb, H. H.; Piantavigna, S.; Howard, C. B.; Nouwens, A.; Mahler, S. M.; Middelberg, A. J.; He, L.; Holt, S.; Sainsbury, F., Insights into the interfacial structure-function of poly(ethylene glycol)-decorated peptide-stabilised nanoscale emulsions. Soft Matter 2017, 13, 7953-7961. 
19. Herman, S.; Hooftman, G.; Schacht, E., Poly(Ethylene Glycol) with Reactive Endgroups .1. Modification of Proteins. J. Bioact. Compat. Polym. 1995, 10, 145-187.

20. Jokerst, J. V.; Lobovkina, T.; Zare, R. N.; Gambhir, S. S., Nanoparticle PEGylation for imaging and therapy. Nanomedicine (London, U. K.) 2011, 6, 715-728.

21. Mateo, C.; Lombardero, J.; Moreno, E.; Morales, A.; Bombino, G.; Coloma, J.; Wims, L.; Morrison, S. L.; Pérez, R., Removal of amphipathic epitopes from genetically engineered antibodies: production of modified immunoglobulins with reduced immunogenicity. Hybridoma 2000, 19, 463-471.

22. Fee, C. J.; Van Alstine, J. M., PEG-proteins: Reaction engineering and separation issues. Chem. Eng. Sci. 2006, 61, 924-939.

23. Stephanopoulos, N.; Francis, M. B., Choosing an effective protein bioconjugation strategy. Nat. Chem. Biol. 2011, 7, 876-84.

24. Nair, D. P.; Podgórski, M.; Chatani, S.; Gong, T.; Xi, W.; Fenoli, C. R.; Bowman, C. N., The Thiol-Michael Addition Click Reaction: A Powerful and Widely Used Tool in Materials Chemistry. Chem. Mater. 2014, 26, 724-744.

25. Tcholakova, S.; Denkov, N. D.; Ivanov, I. B.; Campbell, B., Coalescence stability of emulsions containing globular milk proteins. Adv. Colloid Interface Sci. 2006, 123-126, 259-293.

26. Sainsbury, F.; Zeng, B.; Middelberg, A. P., Towards designer nanoemulsions for precision delivery of therapeutics. Curr. Opin. Chem. Eng. 2014, 4, 11-17.

27. Malcolm, A. S.; Dexter, A. F.; Katakdhond, J. A.; Karakashev, S. I.; Nguyen, A. V.; Middelberg, A. P., Tuneable control of interfacial rheology and emulsion coalescence. ChemPhysChem 2009, 10, 778-781.

28. Muller, E.; Josic, D.; Schroder, T.; Moosmann, A., Solubility and binding properties of PEGylated lysozyme derivatives with increasing molecular weight on hydrophobicinteraction chromatographic resins. J. Chromatogr. A 2010, 1217, 4696-703. 
TOC Graphic

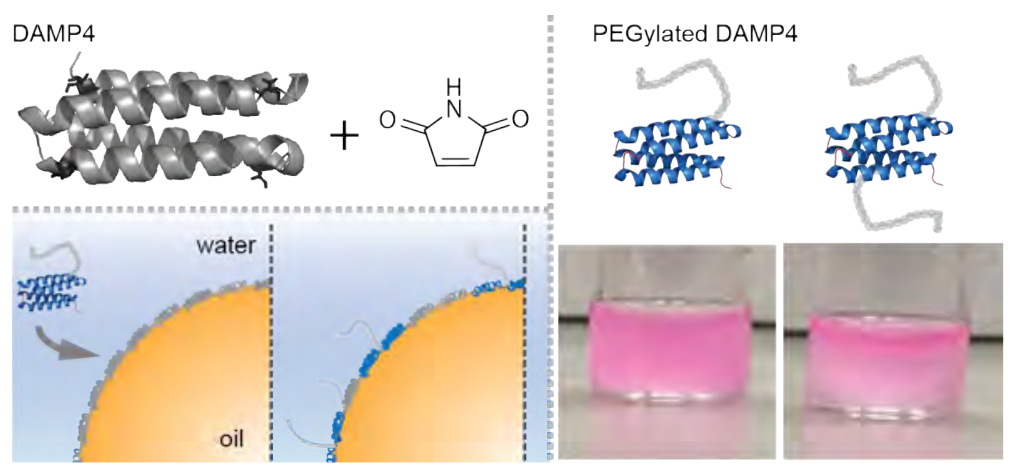

